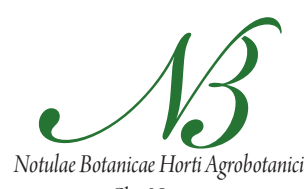

\title{
Influence of Substrate and Fertilization on Growth and Development of Iris adriatica
}

\author{
Ines HAN DOVEDAN ${ }^{1}$, Sanja MORIĆ ${ }^{1}$, Zoran ŠINDRAK², Ivana \\ CEROVSKI ${ }^{1}$, Lepomir ČOGA $^{3}$, Ivan MUSTAĆ ${ }^{4}$, Miroslav POJE $^{1}$ \\ ${ }^{1}$ University of Zagreb, Faculty of Agriculture, Department of Ornamental Plants, Landscape Architecture \\ and Garden Art, Svetosimunska cesta 25, 10000 Zagreb,Croatia; smoric@agr.hr \\ ${ }^{2}$ University of Zagreb, Faculty of Agriculture, Department of Pomology, Svetosimunska cesta 25, 10000 Zagreb, Croatia \\ ${ }^{3}$ University of Zagreb, Faculty of Agriculture, Department of Plant Nutrition, Svetosimunska cesta 25, 10000 Zagreb, Croatia \\ ${ }^{4}$ University of Zagreb, Faculty of Agriculture, Department of Soil Amelioration, Svetošimunska cesta 25, 10000 Zagreb, Croatia
}

\begin{abstract}
Iris adriatica is indigenous to Mediterranean part of Croatia. It is an attractive plant and has opportunities for introduction into horticulture and the ornamental plant market. Research was conducted in the experimental garden of the Faculty of Agriculture, University of Zagreb, to assess the possibility of growing this species as a pot plant. Research included 360 potted plants placed as randomized block design experiment. The goal of this research was to determine influence of chosen substrates and fertilizers, as well as to explore growth and development dynamic of the species cultivated outdoors from June till August 2010. The following characteristics were measured: plants height, number of leaves, and number of rosettes. Biometric analysis showed that the development of Iris adriatica was significantly influenced only by substrate, while fertilization and interaction of substrate and fertilization showed no significant impact on examined plants characteristics $(p \leq 0.05)$.
\end{abstract}

Keywords: Croatian flora, endemic plant species, introduction, ornamental horticulture, pot plant

\section{Introduction}

Croatian endemic species Iris adriatica Trinajstić ex Mitić is a rhizomatic perennial plant from the family of Iridaceae (Fig. 1). Distribution of the species is endemic to Mediterranean region of Croatia. It is decorative due to its small habit (height up to $10 \mathrm{~cm}$ ) and very attractive yellow and purple flowers which appear in June and July. It is mostly propagated by rhizome division but seed propagation is also possible, although much more difficult, Kereša et al. (2009) recommended micropropagation for commercial production as an ornamental plant.

Iris adriatica is included in Croatian Red Book of plant species (Nikolić and Topić, 2005; Šugar, 1994) where it is listed as endangered and almost endangered species with limited habitat and small population, some places completely vanished or decreased in number. Main causes of decreased population are urbanization and natural succession. The species is protected in Croatia, according to the Ordinance on Designating Wild Taxa Protected and Strictly Protected (NN 7/06) and has not yet been introduced into horticultural production so it would be a novel on ornamental plant market (Halevy, 1999).

Introduction into horticultural production means, except of broadening ornamental plants offer, also ex-situ protection of plant species which are otherwise destroyed at their natural habitat by excessive collecting, often due to its attractive appearances (Hajoš et al., 2003).
The goal of this research was to assess the possibility of growing this species as a pot plant by examining influence of chosen substrates and fertilizers on growth and development of the species outdoors over a period of six weeks.

\section{Materials and methods}

Experiment was conducted with 360 vegetatively propagated plants of species Iris adriatica in the experimental garden of Department of Ornamental Plants, Landscape Architecture and Garden Art at the Faculty of Agriculture, University of Zagreb, Croatia, from June $24^{\text {th }}$ to August $25^{\text {th }} 2010$. To ensure uniform growth conditions plants were propagated by rhizome division and planted into plastic pots ( $14 \mathrm{~cm}$ radius and $1.1 \mathrm{~L}$ volume). In the experiment two types of substrates were used (Tab. 1).

In experiment a specialized liquid fertilizer (factor $\mathrm{F}$ ) for house and balcony plants was used. This fertilizer is intended to be used on flowering species because in addition to main macrofertilizers in composition 6-7-8 (6\% nitrogen as amide, nitrate and ammoniac, $7 \%$ phosphorus solvable in water $\mathrm{P}_{2} \mathrm{O}_{5}$, and $8 \%$ potassium solvable in water $\left.\mathrm{K}_{2} \mathrm{O}\right)$. The fertilizer also contains the most important microfertilizers which are necessary to achieve quality of flowers and induce vegetative growth. The fertilizer also contains microelements $\mathrm{Fe}, \mathrm{Cu}, \mathrm{Zn}$ and $\mathrm{B}$. Tab. 2 shows monthly values of air temperature, duration of sunlight, 


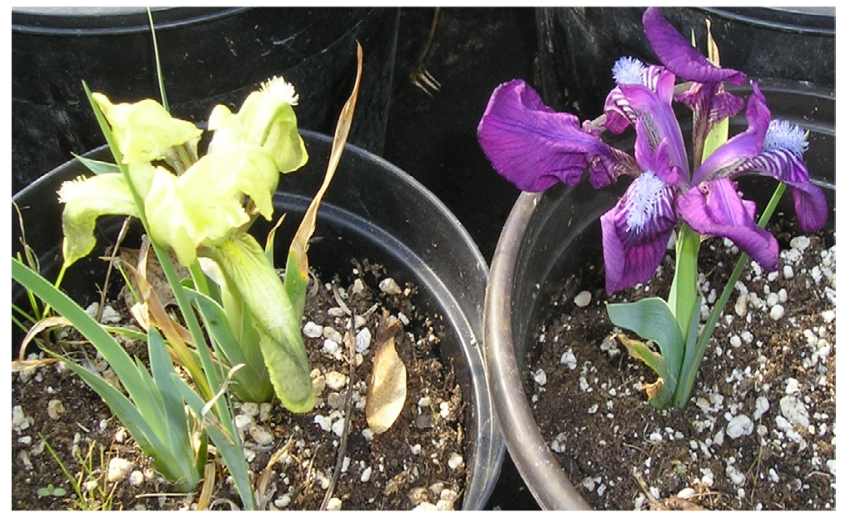

Fig. 1. Iris adriatica Trinajstić ex Mitić, photographed by Morić, 2010

precipitation, number of sunny, foggy and rainy days in Zagreb for the period in which experiment was conducted (source: Croatian Meteorological and Hydrological Service).

This two factorial experiment (factor S-substrate, $\mathrm{F}$ factor-fertilization) is set according to the randomized block design with five blocks. Both factors had two levels and there were four combinations: $S_{1} F_{0}-$ Substrate 1 , no fertilizer; $S_{1} F_{1}$-Substrate $1,1 \%$ solution of fertilizer; $S_{2} F_{0}$ Substrate 2, no fertilizer; $S_{2} F_{1}$-Substrate 2, $1 \%$ solution of fertilizer.

Tab. 1. Structure of substrates (factor $S$ ) used in the experiment

\begin{tabular}{|c|c|c|c|}
\hline Structure & & $\begin{array}{c}\text { Substrate } 1 \\
\left(\text { level } S_{1}\right)\end{array}$ & $\begin{array}{c}\text { Substrate } 2 \\
\left(\text { level } S_{2}\right)\end{array}$ \\
\hline \multirow{3}{*}{ Composition (\%): } & white peat & 40 & 40 \\
\hline & mixed peat & 56 & 56 \\
\hline & clay & 4 & 4 \\
\hline Organic matter (\%) & & 80 & 58.5 \\
\hline $\mathrm{pH}\left(\mathrm{H}_{2} \mathrm{O}\right)$ & & $6.5-7$ & $6.5-7$ \\
\hline \multirow{6}{*}{$\begin{array}{l}\text { Macrofertilizer } \\
\text { concentrate } \\
\left(\mathrm{mg} \mathrm{L}^{-1}\right):\end{array}$} & $\mathrm{N}$ & 135 & 200 \\
\hline & $\mathrm{NH}_{4}-\mathrm{N}$ & 90 & 120 \\
\hline & $\mathrm{NO}_{3}-\mathrm{N}$ & 45 & 80 \\
\hline & $\mathrm{P}_{2} \mathrm{O}_{5}$ & 137 & 150 \\
\hline & $\mathrm{K}_{2} \mathrm{O}$ & 200 & 289 \\
\hline & $\mathrm{Mg}$ & 60 & 90.1 \\
\hline Moisture (\%) & & $65-75$ & $65-75$ \\
\hline $\begin{array}{c}\text { Dry matter (DM), } \\
105^{\circ} \mathrm{C}(\%)\end{array}$ & & $30-40$ & $40-50$ \\
\hline \multirow{11}{*}{$\begin{array}{l}\text { Total heavy metals } \\
\left(\mathrm{mg} \mathrm{kg}^{-1} \mathrm{DM}\right):\end{array}$} & $\mathrm{Mn}$ & 177.2 & 188.1 \\
\hline & $\mathrm{Zn}$ & 18 & 26.3 \\
\hline & $\mathrm{Cu}$ & 25.48 & 19.1 \\
\hline & $\mathrm{Pb}$ & 12.66 & 11.56 \\
\hline & $\mathrm{Cd}$ & 0.88 & 0.7 \\
\hline & $\mathrm{Cr}$ & 1.1 & 0.9 \\
\hline & $\mathrm{Ni}$ & 6.71 & 4.3 \\
\hline & As & 1.1 & 1.1 \\
\hline & $\mathrm{Hg}$ & in traces & in traces \\
\hline & Mo & 0.101 & 0.065 \\
\hline & Co & 2.1 & 1.1 \\
\hline
\end{tabular}

In the experiment the following characteristics were measured:-plants height (measured from root neck to the highest part of the plant),-maximum number of leaves per plant,-number of rosettes counted on four dates: July $14^{\text {th }}$, July $28^{\text {th }}$, August $11^{\text {th }}$ and August $25^{\text {th }}$. Solution of fertil$\operatorname{izer}\left(10 \mathrm{ml} \mathrm{L}^{-1} \mathrm{H}_{2} \mathrm{O}\right)$ was applied on three dates: July $15^{\text {th }}$, July $29^{\text {th }}$, August $12^{\text {th }}$ i.e. every 14 days with $0.25 \mathrm{dL}$ of solution per pot.

\section{Statistical_analysis}

Analysis of variance (ANOVA) of two factorial experiment (Gomez and Gomez, 1984) was performed according to randomized block experimental design with five blocks using the commercial software SAS Ver. 9.1.' Values are presented as a means of five replications.

\section{Results and discussion}

Plants grown in Substrate 1 were significantly shorter (in average $2 \mathrm{~cm}$ ) than plants grown in Substrate 2, which may be related to the level of nutrients in substrate. Plants grown in supstrate containing a higher level of nutrients (especially nitrogen) had a stronger vegetative growth, and a smaller number of rosettes (Tab. 3 ). In contrast to the plant height, the number of rosettes per plant was higher in plants grown in Substrate 1 (Tab. 5). There was no statistically significant influence of substrate on number of leaves per plant, except in measurement on July $28^{\text {th }}$, although a higher number of leaves were measured on plants grown in Substrate 2 (Tab. 4). All the results indicate that plants grown on Substrate 1 had a more compact growth which is highly desirable in growing this species as a flowering pot plant.

Balanced fertilization is very important for growth and development of flowering pot plants whereby it is relevant that certain amount of nutrition supply is available to the plant in different stages of growth.

Only basic guidelines for amounts of necessary nutrition solutions are available for most ornamental plants, except for some important species which are grown in large scale (Finck, 1982; Hershey and Paul, 1981; King et al.,

Tab. 2. Meteorological values for Zagreb in June, July and August 2010

\begin{tabular}{ccccc}
\hline \multicolumn{2}{c}{ Zagreb (2010) } & Jun & Jul & Aug \\
\hline \multirow{2}{*}{$\begin{array}{c}\text { Air temperature } \\
\left({ }^{\circ} \mathrm{C}\right)\end{array}$} & mean & 19.1 & 20.8 & 20 \\
& aps. max. & 37.6 & 40.4 & 39.8 \\
& aps. min. & 2.5 & 5.4 & 3.7 \\
\hline Sunlight (in hours) & & 243.7 & 281 & 256 \\
\hline $\begin{array}{c}\text { Precipitation } \\
(\mathrm{mm})\end{array}$ & & 96 & 81.6 & 89.3 \\
\hline \multirow{2}{*}{$\begin{array}{c}\text { Number of days } \\
\end{array}$} & funny & 3 & 7 & 8 \\
& foggy & 1 & 0 & 1 \\
\hline & wainy & 13 & 11 & 10 \\
& hot & 15 & 22 & 20 \\
\hline
\end{tabular}


214

Tab. 3. Substrate and fertilization influence on plant height of Iris adriatica. Partially outline of the ANOVA for plant height characteristic; mean values for main effects levels and their combinations are listed on the right side

\begin{tabular}{|c|c|c|c|c|c|c|c|c|c|}
\hline Source of variation & Degree of freedom & Date & Computed F & $\operatorname{Pr}>F$ & \multicolumn{5}{|c|}{ Plant height $(\mathrm{cm})$} \\
\hline \multirow{4}{*}{$\begin{array}{l}\text { Treatment } \\
\text { combinations }\end{array}$} & \multirow{4}{*}{3} & Jul. $14^{\text {th }}$ & $2.33^{\text {n.s. }}$ & 0.1259 & & & & & \\
\hline & & Jul. $28^{\text {th }}$ & $6.85^{* *}$ & 0.0061 & & & & & \\
\hline & & Aug. $11^{\text {th }}$ & $8.81^{* *}$ & 0.0023 & & & & & \\
\hline & & Aug. $25^{\text {th }}$ & $3.49^{*}$ & 0.0500 & & & & & \\
\hline \multirow{5}{*}{ Substrate $(S)$} & \multirow{5}{*}{ (1) } & & & & \multicolumn{2}{|c|}{$S_{1}$} & \multicolumn{2}{|c|}{$\mathrm{S}_{2}$} & \\
\hline & & Jul. $14^{\text {th }}$ & $4.94^{\text {n.s }}$ & 0.0462 & \multicolumn{2}{|c|}{9.9} & \multicolumn{2}{|c|}{11.1} & \\
\hline & & Jul. $28^{\text {th }}$ & $20.37^{* *}$ & 0.0007 & \multicolumn{2}{|c|}{9.5} & \multicolumn{2}{|c|}{12.0} & ** \\
\hline & & Aug. $11^{\text {th }}$ & $25.69^{* *}$ & 0.0003 & \multicolumn{2}{|c|}{10.2} & \multicolumn{2}{|c|}{12.8} & ** \\
\hline & & Aug. $25^{\text {th }}$ & $10.15^{* *}$ & 0.0078 & \multicolumn{2}{|c|}{10.8} & \multicolumn{2}{|c|}{12.9} & ** \\
\hline \multirow{5}{*}{ Fertilization $(\mathrm{F})$} & \multirow{5}{*}{ (1) } & & & & \multicolumn{2}{|c|}{$\mathrm{F}_{1}$} & \multicolumn{2}{|c|}{$\mathrm{F}_{0}$} & \\
\hline & & Jul. $14^{\text {th }}$ & $1.85^{\mathrm{n} . \mathrm{s}}$ & 0.1985 & \multicolumn{2}{|c|}{10.2} & \multicolumn{2}{|c|}{10.9} & \\
\hline & & Jul. $28^{\text {th }}$ & $0.19^{\text {n.s. }}$ & 0.6689 & \multicolumn{2}{|c|}{10.6} & \multicolumn{2}{|c|}{10.9} & \\
\hline & & Aug. $11^{\text {th }}$ & $0.00^{\mathrm{n} . \mathrm{s}}$ & 0.9448 & \multicolumn{2}{|c|}{11.5} & \multicolumn{2}{|c|}{11.5} & \\
\hline & & Aug. $25^{\text {th }}$ & $0.26^{\mathrm{ns.} .}$ & 0.6175 & \multicolumn{2}{|c|}{11.7} & \multicolumn{2}{|c|}{12.0} & \\
\hline \multirow{5}{*}{ Interaction $(\mathrm{SxF})$} & \multirow{5}{*}{ (1) } & & & & $\mathrm{S}_{1} \mathrm{~F}_{1}$ & $\mathrm{~S}_{1} \mathrm{~F}_{0}$ & $\mathrm{~S}_{2} \mathrm{~F}_{1}$ & $\mathrm{~S}_{2} \mathrm{~F}_{0}$ & \\
\hline & & Jul. $14^{\text {th }}$ & $0.20^{\mathrm{n} . \mathrm{s}}$ & 0.6625 & 9.7 & 10.2 & 10.6 & 11.6 & \\
\hline & & Jul. $28^{\text {th }}$ & $0.00^{\mathrm{n} . \mathrm{s}}$ & 0.9916 & 9.4 & 9.6 & 11.9 & 12.1 & \\
\hline & & Aug. $11^{\text {th }}$ & $0.73^{\mathrm{n} . \mathrm{s}}$ & 0.4109 & 10.4 & 10.0 & 12.6 & 13.0 & \\
\hline & & Aug. $25^{\text {th }}$ & $0.01^{\text {n.s. }}$ & 0.9386 & 10.7 & 11.0 & 12.7 & 13.1 & \\
\hline
\end{tabular}

n.s.-not significant; ${ }^{* *}$-significant at $p \leq 0.01 ;{ }^{*}$-significant at $p \leq 0.05 ; \mathrm{S}_{1}=$ Substrate $1 ; \mathrm{S}_{2}=$ Substrate $2 ; \mathrm{F}_{1}=$ fertilized; $\mathrm{F}_{0}=$ not fertilized; Int. $\mathrm{S} \times \mathrm{F}=$ interaction substratexfertilization

1995; Macz et al., 2001). Regarding the fact that growing of species of genus Iris (Armitage, 1995; Ganslmeier and Henseler, 1985; Nau, 1996) requires substrate rich in nutrition solutions, two commercial substrates different in organic matter percentage and nutritions concentration were used in this experiment. According to research conducted by Vršek et al. (2004) natural habitat of species $I$. adriatica is rich in humus (9.83 to $11.38 \%$ ), contains low levels of plant available phosphorus (1.75 to $2.18 \mathrm{mg} \mathrm{P}$ $\mathrm{kg}^{-1}$ ) while potassium and nitrogen are highly available to the plant (190.92-315.44 $\mathrm{mg} \mathrm{K} \mathrm{kg}^{-1}$ and $45.16-67.74 \mathrm{mg}$ $\left.\mathrm{Nkg}^{-1}\right)$.

Marschner (1995) states that many perennials have growth adapted to soils with low levels of nutrition solu-

Tab. 4. Substrate and fertilization influence on number of leaves per plant of Iris adriatica. Partially outline of the ANOVA for number of leaves characteristic; mean values for main effects levels and their combinations are listed on the right side

\begin{tabular}{|c|c|c|c|c|c|c|c|c|c|}
\hline Source of variation & Degree of freedom & Date & Computed F & $\operatorname{Pr}>F$ & \multicolumn{5}{|c|}{ Number of leaves per plant } \\
\hline \multirow{4}{*}{$\begin{array}{l}\text { Treatment } \\
\text { combinations }\end{array}$} & \multirow{4}{*}{0} & Jul. $14^{\text {th }}$ & $2.78^{\text {n.s }}$ & 0.0869 & & & & & \\
\hline & & Jul. $28^{\text {th }}$ & $8.20^{* *}$ & 0.0031 & & & & & \\
\hline & & Aug. $11^{\text {th }}$ & $2.69^{\text {n.s. }}$ & 0.0936 & & & & & \\
\hline & & Aug. $25^{\text {th }}$ & $1.49^{\mathrm{ns}}$ & 0.0936 & & & & & \\
\hline \multirow{5}{*}{ Substrate (S) } & \multirow{5}{*}{ (1) } & & & & \multicolumn{2}{|c|}{$\mathrm{S}_{1}$} & \multicolumn{2}{|c|}{$\mathrm{S}_{2}$} & \\
\hline & & Jul. $14^{\text {th }}$ & $6.58^{\text {n.s. }}$ & 0.0248 & \multicolumn{2}{|c|}{4.0} & \multicolumn{2}{|c|}{4.3} & \\
\hline & & Jul. $28^{\text {th }}$ & $24.26^{* *}$ & 0.0004 & \multicolumn{2}{|c|}{4.1} & \multicolumn{2}{|c|}{4.9} & ** \\
\hline & & Aug. $11^{\text {th }}$ & $7.53^{\text {n.s. }}$ & 0.0178 & \multicolumn{2}{|c|}{4.8} & \multicolumn{2}{|c|}{5.4} & \\
\hline & & Aug. $25^{\text {th }}$ & $4.13^{\text {n.s. }}$ & 0.0649 & \multicolumn{2}{|c|}{5.2} & \multicolumn{2}{|c|}{5.6} & \\
\hline \multirow{5}{*}{ Fertilization (F) } & \multirow{5}{*}{ (1) } & & & & \multicolumn{2}{|c|}{$\mathrm{F}_{1}$} & \multicolumn{2}{|c|}{$\mathrm{F}_{0}$} & \\
\hline & & Jul. $14^{\text {th }}$ & $1.50^{\mathrm{ns}}$ & 0.2434 & \multicolumn{2}{|c|}{4.1} & \multicolumn{2}{|c|}{4.2} & \\
\hline & & Jul. $28^{\text {th }}$ & $0.31^{\mathrm{n} . \mathrm{s}}$ & 0.5886 & \multicolumn{2}{|c|}{4.6} & \multicolumn{2}{|c|}{4.5} & \\
\hline & & Aug. $11^{\text {th }}$ & $0.53^{\mathrm{n} . \mathrm{s}}$ & 0.4821 & \multicolumn{2}{|c|}{5.2} & \multicolumn{2}{|c|}{5.0} & \\
\hline & & Aug. $25^{\text {th }}$ & $0.01^{\text {n.s. }}$ & 0.9314 & \multicolumn{2}{|c|}{5.4} & \multicolumn{2}{|c|}{5.4} & \\
\hline \multirow{5}{*}{ Interaction $(\mathrm{SxF})$} & \multirow{5}{*}{ (1) } & & & & $\mathrm{S}_{1} \mathrm{~F}_{1}$ & $\mathrm{~S}_{1} \mathrm{~F}_{0}$ & $\mathrm{~S}_{2} \mathrm{~F}_{1}$ & $\mathrm{~S}_{2} \mathrm{~F}_{0}$ & \\
\hline & & Jul. $14^{\text {th }}$ & $0.25^{\mathrm{n} . \mathrm{s}}$ & 0.6274 & 3.9 & 4.1 & 4.3 & 4.4 & \\
\hline & & Jul. $28^{\text {th }}$ & $0.02^{\mathrm{ns}}$ & 0.8918 & 4.2 & 4.1 & 5.0 & 4.9 & \\
\hline & & Aug. $11^{\text {th }}$ & $0.00^{\mathrm{ns}}$ & 0.9637 & 4.9 & 4.7 & 5.5 & 5.3 & \\
\hline & & Aug. $25^{\text {th }}$ & $0.32^{\text {n.s. }}$ & 0.5815 & 5.2 & 5.1 & 5.5 & 5.7 & \\
\hline
\end{tabular}

Note: n.s.-not significant; ${ }^{* *}$-significant at $p \leq 0.01 ;{ }^{*}$-significant at $p \leq 0.05 ; \mathrm{S}_{1}=$ Substrate $1 ; \mathrm{S}_{2}=$ Substrate $2 ; \mathrm{F}_{1}=$ fertilized; $\mathrm{F}_{0}=$ not fertilized; Int. $S \times \mathrm{F}=$ interaction substratexfertilization 
Tab. 5. Substrate and fertilization influence on number of rosettes per plant of Iris adriatica. Partially outline of the ANOVA for number of rosettes; mean values for main effects levels and their combinations are listed on the right side

\begin{tabular}{|c|c|c|c|c|c|c|c|c|c|}
\hline Source of variation & Degree of freedom & Date & Computed F & $\operatorname{Pr}>F$ & \multicolumn{5}{|c|}{ Number of rosettes per plant } \\
\hline \multirow{4}{*}{$\begin{array}{l}\text { Treatment } \\
\text { combinations }\end{array}$} & \multirow{4}{*}{3} & Jul. $14^{\text {th }}$ & $14.85^{* *}$ & 0.0002 & & & & & \\
\hline & & Jul. $28^{\text {th }}$ & $10.69^{* *}$ & 0.0010 & & & & & \\
\hline & & Aug. $11^{\text {th }}$ & $12.52^{* *}$ & 0.0005 & & & & & \\
\hline & & Aug. $25^{\text {th }}$ & $6.84^{* *}$ & 0.0061 & & & & & \\
\hline \multirow{5}{*}{ Substrate $(S)$} & \multirow{5}{*}{$(1)$} & & & & \multicolumn{2}{|c|}{$S_{1}$} & \multicolumn{2}{|c|}{$\mathrm{S}_{2}$} & \\
\hline & & Jul. $14^{\text {th }}$ & $43.55^{* *}$ & $<0.0001$ & \multicolumn{2}{|c|}{1.96} & \multicolumn{2}{|c|}{1.31} & ** \\
\hline & & Jul. $28^{\text {th }}$ & $31.14^{* *}$ & 0.0001 & \multicolumn{2}{|c|}{1.88} & \multicolumn{2}{|c|}{1.37} & ** \\
\hline & & Aug. $11^{\text {th }}$ & $35.05^{* *}$ & $<0.0001$ & \multicolumn{2}{|c|}{1.96} & \multicolumn{2}{|c|}{1.49} & ** \\
\hline & & Aug. $25^{\text {th }}$ & $18.23^{* *}$ & 0.0011 & \multicolumn{2}{|c|}{2.05} & \multicolumn{2}{|c|}{1.55} & ** \\
\hline \multirow{5}{*}{ Fertilization $(\mathrm{F})$} & \multirow{5}{*}{$(1)$} & & & & \multicolumn{2}{|c|}{$\mathrm{F}_{1}$} & \multicolumn{2}{|c|}{$\mathrm{F}_{0}$} & \\
\hline & & Jul. $14^{\text {th }}$ & $0.00^{\text {n.s. }}$ & 1.0000 & \multicolumn{2}{|c|}{1.64} & \multicolumn{2}{|c|}{1.64} & \\
\hline & & Jul. $28^{\text {th }}$ & $0.29^{\text {n.s. }}$ & 0.6000 & \multicolumn{2}{|c|}{1.65} & \multicolumn{2}{|c|}{1.37} & \\
\hline & & Aug. $11^{\text {th }}$ & $1.45^{\mathrm{n} . \mathrm{s}}$ & 0.2517 & \multicolumn{2}{|c|}{1.77} & \multicolumn{2}{|c|}{1.67} & \\
\hline & & Aug. $25^{\text {th }}$ & $0.01^{\text {n.s. }}$ & 0.9331 & \multicolumn{2}{|c|}{1.80} & \multicolumn{2}{|c|}{1.79} & \\
\hline \multirow{5}{*}{ Interaction $(\mathrm{SxF})$} & \multirow{5}{*}{ (1) } & & & & $S_{1} F_{1}$ & $\mathrm{~S}_{1} \mathrm{~F}_{0}$ & $\mathrm{~S}_{2} \mathrm{~F}_{1}$ & $\mathrm{~S}_{2} \mathrm{~F}_{0}$ & \\
\hline & & Jul. $14^{\text {th }}$ & $1.01^{\text {n.s. }}$ & 0.3351 & 1.91 & 2.01 & 1.36 & 1.26 & \\
\hline & & Jul. $28^{\text {th }}$ & $0.64^{\mathrm{ns.s}}$ & 0.4409 & 1.87 & 1.90 & 1.43 & 1.30 & \\
\hline & & Aug. $11^{\text {th }}$ & $1.06^{\mathrm{n} . \mathrm{s} .}$ & 0.3240 & 1.96 & 1.95 & 1.57 & 1.40 & \\
\hline & & Aug. $25^{\text {th }}$ & $2.28^{\text {n.s. }}$ & 0.1572 & 1.96 & 2.13 & 1.64 & 1.45 & \\
\hline
\end{tabular}

Note: n.s.-not significant; ${ }^{* *}$-significant at $p \leq 0.01 ;{ }^{*}$-significant at $p \leq 0.05 ; \mathrm{S}_{1}=$ Substrate $1 ; \mathrm{S}_{2}=$ Substrate $2 ; \mathrm{F}_{1}=$ fertilized; $\mathrm{F}_{0}=$ not fertilized; Int. $\mathrm{S} \times \mathrm{F}=$ interaction substrate $\times$ fertilization

tions, and that they continue to grow without showing any visual symptoms of deficiency.

Finck (1982) indicates that efforts to achieve optimal levels of nutrition solutions in soil during plant growth often result in exceeding of optimal levels and in danger of over fertilization.

Biometrical analysis clearly shows that the only factor with significant influence on growth of plant species Iris adriatica was substrate, while fertilization and interaction of substrate and fertilization did not show any significant influence in measured plants characteristics (with $\mathrm{P} \leq 0.05$ ). In this particular case this means that fertilization can be left out which decreases the costs of plants production. This research confirms it is possible to grow plant species Iris adriatica as a flowering pot plant.

\section{References}

Armitage AM (1995). Speciality Cut Flowers, $1^{\text {st }}$ Ed. Timber Press, Portland, Oregon.

Finck A (1982). Fertilizers and Fertilization, Verlag Chemie, Weinheim.

Ganslmeier H, K Henseler (1985). Schnittstauden, Verlag Eugen Ulmer, Stuttgart.

Gomez KA, Gomez AA (1984). Statistical Procedures for Agricultural Research, $2^{\text {nd }}$ Ed. John Wiley and Sons, Inc., New York.

Hajoš D, Vršek I, Karlović K, Židovec V, Morić S (2003). Commercial cultivation of indigenous plant species, a measure of ex-situ conservation (in Croatian). Sjemenarstvo 20:37-45.

Halevy AH (1999). Ornamentals: Where diversity is king-the Israeli experience. Perspectives on new crops and new uses.
ASHS Press 404-406.

Hershey DR, Paul JL (1981). Critical foliar levels of potassium in pot chrysanthemum. Hort Sci 16(2):220-222.

Kereša S, Mihovilović A, Ćurković-Perica M, Mitić B, Barić M, Vršek I, Marchetti S (2009). In vitro regeneration of the Croatian endemic species Iris adriatica Trinajstić ex Mitić. Acta Biologica Cracoviensia Series Botanica 51(2):7-12.

King JJ, Peterson LA, Stimart DP (1995). Ammonium and nitrate uptake through development in Dendranthema grandfolium. Hort Sci 30(3):499-503.

Macz O, Paparozzi ET, Stroup W (2001). Effect of nitrogen and sulfur applications on pot chrysanthemum production and postharvest performance. I. leaf nitrogen and sulfur concentrations. Journal of Plant Nutrition 24(1):111-129.

Marschner H (1995). Mineral Nutrition of Higher Plants, $2^{\text {nd }}$ Ed. Academic Press, London.

Nau J (1996). Ball Perennial Manual, $1^{\text {st }}$ Ed. Ball Publishing, Batavia, Illinois.

Nikolić T, Topić J (2005). Red Book of Vascular Flora of Croatia, Categories EX, RE, CR, EN and VU, Ministry of culture, State Institute for Nature Protection, Zagreb.

SAS Institute Inc. (2004). SAS/STAT Software, Version 9.1., User's Guide, SAS Institute, Cary, NC.

Šugar I (1994). Red Book of Plant Species of Croatia, Ministry of Environmental Protection, Physical Planning and Construction, Institute for Nature Protection, Zagreb.

Vršek I, Mitić B, Bujan M, Čoga L, Milović M, Richter M (2004). Iris adriatica Trinajstić ex Mitić, potential plant suitable for cultivation in pots, $173-174$ p. In: Mitić B, Šoštarić R (Eds.). Book of abstracts. Prvi hrvatski botanički simpozij s međunarodnim sudjelovanjem. Hrvatsko botaničko društvo, Zagreb. 